\title{
Atividade artística para avaliar o consumo alimentar e os conhecimentos de nutrição em crianças com excesso de peso
}

\section{Artistic activity to evaluate dietary intake and nutritional knowledge in overweight children}

\author{
Caroline Andrade Vinhal ${ }^{1}$ (D) , Luciane Cristina de Oliveira Lisboa ${ }^{2}$ (1) , Martha Elisa Ferreira de Almeida ${ }^{2}$ (1)
}

1. Bacharel em Nutrição pela Universidade Federal de Viçosa (UFV), Campus Rio Paranaíba, MG, Brasil. 2. Docente do Instituto de Ciências Biológicas e da Saúde, Universidade Federal de Viçosa, Campus Rio Paranaíba, Rio Paranaíba, MG, Brasil.

\begin{abstract}
Resumo
Objetivo: avaliar o consumo alimentar e os conhecimentos em nutrição por meio de atividades artísticas, em crianças com excesso de peso antes e depois de atividades de educação alimentar e nutricional. Métodos: utilizaram-se algumas atividades de educação alimentar e nutricional como uma gincana para avaliar os órgãos dos sentidos, a pirâmide alimentar e um semáforo, bem como a montagem artística de dois pratos referentes a uma refeição. Analisaramse os dados da gincana pelo Teste do $\chi 2$ a $5 \%$ de significância. Resultados: o feijão foi o único alimento que apresentou diferença estatística (p<0,05) em relação ao tato. Todos os avaliados colocaram os alimentos corretamente na pirâmide alimentar, e, na prova do semáforo, os meninos apresentaram mais acertos. 0 feijão permaneceu no prato de todos os participantes depois das atividades trabalhadas, havendo a retirada do arroz pelas crianças obesas e os meninos com sobrepeso. Em alguns pratos, houve a retirada das carnes, dos ovos, das frutas e hortaliças, e outros permaneceram com os alimentos ricos em óleos e gorduras, açúcares e doces. Conclusão: as atividades de educação alimentar e nutricional melhoraram a percepção quanto à alimentação, e, consequentemente, o consumo alimentar expresso por meio da atividade artística, fazendo-se necessárias outras ações para trabalhar a ingestão de açúcares e doces, óleos e gorduras e refrigerantes.
\end{abstract}

Palavras-chave: Lúdico. Educação alimentar e nutricional. Obesidade.

\begin{abstract}
Objective: to evaluate food consumption and knowledge in nutrition by means of artistic activities in overweight children before and after food and nutrition in education activities. Methods: was used some food and nutrition education activities as a gymkhana to evaluate the sense of the organs, the food pyramid and a traffic light, as well as the artistic assembling of two dishes referring to a meal. The data of the gymkhana were analyzed by the $\chi 2$ Test at $5 \%$ of significance. Results: beans were the only food that presented statistical difference $(p<0.05)$ in relation to touch. All the evaluated ones placed the foods correctly in the food pyramid, and in the test of the semaphore the boys presented more correctness. The beans remained on the plate of all participants after the activities worked, with the withdrawal of rice by obese children and overweight boys. In some dishes there was the withdrawal of meat, eggs, fruits and vegetables, and others remained with foods rich in oils and fats, sugars and sweets. Conclusion: the food and nutritional education activities improved the perception about food, and consequently food consumption expressed by means of artistic activity, requiring other actions to work on the intake of sugars and sweets, oils, fats and soft drinks.
\end{abstract}

Key words: Ludic. Food and nutritional education. Obesity.

\section{INTRODUÇÃO}

O padrão alimentar brasileiro vem sofrendo transformações no decorrer dos anos, o que ocasiona um desequilíbrio no consumo de calorias que excede à quantidade recomendada. A alimentação está diretamente relacionada com a saúde e o bem-estar do indivíduo e, quando apresenta má qualidade, acarretará várias doenças com destaque para a obesidade ${ }^{1}$, que é um problema mundial de saúde que sofre influência dos fatores ambientais, psicológicos e genéticos. Segundo dados da Pesquisa de Orçamentos Familiares (2008-2009), entre as crianças com idade entre 5 a 9 anos, $34,8 \%$ dos meninos e $32 \%$ das meninas apresentavam excesso de peso, bem como 16,6 e $11,8 \%$ possuíam obesidade, respectivamente ${ }^{2}$.
O tratamento e o acompanhamento de crianças com excesso de peso envolvem vários aspectos, sobretudo o comportamental, que enfatiza as mudanças no estilo de vida e na reeducação alimentar e nutricional, que deve ser diferenciada e prazerosa. A dinâmica de grupo é uma técnica educacional utilizada para o desenvolvimento do ensino e aprendizado que possibilita o envolvimento do público pela socialização de vivências, relacionando a teoria à prática, e difere da educação convencional, cuja principal estratégia é a transmissão de informações que, geralmente, apresentam-se insuficientes para mudanças significativas dos hábitos alimentares, devido à 
baixa assimilação do público infantil. Com isso, observa-se um aumento na demanda por abordagens alternativas de educação como o emprego das dinâmicas de grupo, que envolvem a fala, os movimentos corporais e a arte $^{3}$.

O alimento pode ser o elemento principal em uma produção artística e participa ativamente do processo de simbolização, além de uma peculiar capacidade criativa e persuasiva. $\mathrm{Na}$ maioria das vezes, o alimento é enxergado somente do ponto de vista biológico como fonte de energia ao corpo humano, mas a relação homem-alimento tem uma reflexão histórica e cultural. A prática gastronômica, considerada como a "nona arte", aponta as influências culturais individuais de cada prato e revela o alimento como parte integrante da cultura ${ }^{4}$.

A promoção do conhecimento em alimentação e estilo de vida adequada deve receber uma atenção diferenciada. Nesse sentido, a utilização de atividades lúdicas, como dinâmicas de grupo e artes podem contribuir para a obtenção de conhecimentos relacionados à alimentação e à nutrição ${ }^{3}$, especialmente no público infantil, pois o lúdico torna as atividades de educação alimentar nutricional prazerosas, e o indivíduo aprende brincando, fato que poderá incorporar melhor o conhecimento e torná-lo um agente transformador de mudanças na família ${ }^{5}$, uma vez que a condição nutricional tem relação direta com os hábitos familiares.

Cecchetto et al. ${ }^{6}$ destacam que a associação das intervenções lúdicas pode melhorar o conhecimento infantil e o autocuidado, tendo como consequência a prevenção da obesidade. Assim, o objetivo deste estudo foi avaliar o consumo alimentar e os conhecimentos em nutrição por meio de atividades artísticas, em crianças com excesso de peso antes e depois de atividades de educação alimentar e nutricional.

\section{MÉTODOS}

A pesquisa de caráter transversal, experimental e quantitativa, de intervenção foi realizada em outubro de 2014, após a aprovação do projeto pelo Comitê de Ética em Pesquisa da Universidade Federal de Viçosa (UFV), protocolo no. 719.492.

Em uma escola municipal de Carmo do Paranaíba, Minas Gerais, Brasil, havia 183 alunos com a idade entre 6 a 9 anos, e somente 139 obtiveram a autorização dos pais ou cuidadores para participarem do estudo. A partir da autorização dos pais e do assentimento das crianças, obtiveram-se as medidas antropométricas (peso e altura) para o cálculo do Índice de Massa Corporal (IMC) que foi classificado ${ }^{7}$ como critério de seleção da amostra de 57 crianças que apresentaram excesso de peso (sobrepeso ou obesidade). Porém, durante os dias em que aconteceram as atividades, alguns faltaram ou desistiram do estudo, e somente 33 crianças participaram de todas as ações previstas neste estudo.

Após a seleção das crianças, desenvolveram-se as atividades de educação alimentar e nutricional nos dias de segunda-feira (encontros 1, 3 e 5) e quinta-feira (encontros 2, 4 e 6), mas apenas o encontro 7 realizou-se no dia de sexta-feira, após o recreio escolar. Na figura 1, observa-se o detalhamento das atividades realizadas.

Figura 1. Descrição das atividades realizadas em cada encontro com as crianças. Carmo do Paranaíba, Minas Gerais, Brasil.

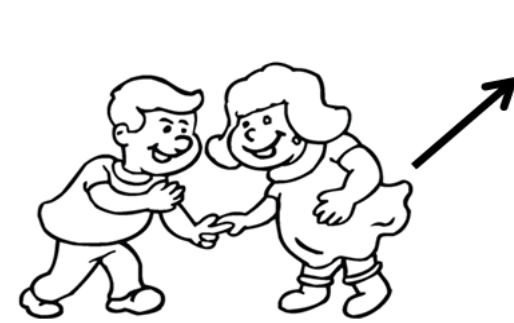

Seleção das crianças

$(n=33)$
Encontro 1: Assinatura do Termo de Assentimento Livre e Esclarecido pelas crianças, e questionamento sobre suas preferências alimentares.

Encontro 2: Elaboração de um prato.

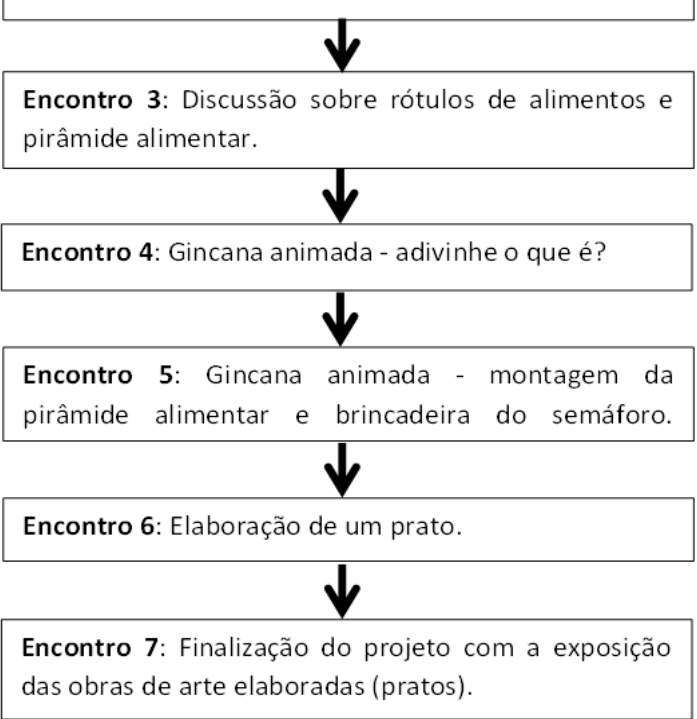


- No encontro 1, explicou-se a proposta do estudo, e as crianças que concordaram em participar assinaram o Termo de Assentimento Livre e Esclarecido. Houve o questionamento às crianças sobre suas preferências alimentares, sem demonstrar nenhuma expressão que pudesse influenciar suas respostas.

- No encontro 2, estimulou-se que as crianças criassem um prato para simular uma refeição habitual (almoço) com os alimentos que foram citados no encontro 1. Utilizaram-se grãos (arroz, milho e feijão), macarrão, e materiais para artesanato como: pratos de papelão, pincéis, cola para artesanato e alimentos modelados em biscuit (ovo, carne de frango, bife de carne bovina, tomate, alface, batata frita, cenoura, beterraba, laranja, banana, maçã, suco, refrigerante, balas e chocolate). No processo de confecção dos pratos, os materiais necessários ficaram dispostos em uma mesa e foram distribuídos, aleatoriamente, aos alunos a seu redor. Os alimentos modelados em biscuit foram previamente elaborados pela equipe de pesquisadoras deste estudo.

- No encontro 3, desenvolveram-se as atividades de educação alimentar e nutricional pela apresentação individual de todos os grupos da pirâmide alimentar infantil ${ }^{8}$. Utilizaram-se rótulos ou alimentos in natura que foram dispostos sobre uma mesa para que as crianças tivessem um maior contato físico e visual. Em cada grupo da pirâmide alimentar, destacaram-se os alimentos que faziam parte, a quantidade que deveria ser ingerida, seus principais nutrientes e a importância nutricional. Comentou-se sobre o histórico de criação da pirâmide alimentar e expôs-se uma pirâmide impressa e colada em uma cartolina.

- No encontro 4, realizou-se a gincana animada adaptada ${ }^{9}$ intitulada - adivinhe o que é? Nessa atividade, cada criança participou, individualmente, das seguintes atividades, utilizando os órgãos dos sentidos (paladar, tato e visão): "experimentando sabores e texturas", "adivinhe o que é?" e "o que você vê?".

A prova "Experimentando sabores e texturas" consistiu em vendar os olhos da criança e colocar o alimento em sua boca para que ela tentasse identificá-lo pelo sabor e a textura. Na prova "Adivinhe o que é?", os olhos da criança foram vendados, e colocou-se um alimento em sua mão para que ela tentasse identificá-lo. Para a prova "O que você vê?", disponibilizaram-se os alimentos em uma mesa, de modo que cada criança ficasse diante deles e falasse o nome de cada um sem tocá-lo. Em todas as avaliações, utilizaram-se os alimentos feijão, alface, cenoura, chuchu e banana", e, na prova do "Experimentando Sabores e Texturas", o feijão, o chuchu e a cenoura foram servidos cozidos em água com a adição de sal. Avaliou-se cada criança nas três provas para cada um dos alimentos citados previamente.

- No encontro 5, ocorreram as provas da "Montagem da pirâmide de alimentos" e "Semáforo dos alimentos". Realizaram-se tais atividades em grupo, dividindo as crianças de acordo com o gênero (meninas versus meninos), para verificar possíveis diferenças na percepção entre elas.

Na prova da "Montagem da pirâmide de alimentos", pregaramse duas pirâmides vazias em papel cartolina no quadro negro, e, ao seu lado, foram colados os recortes de alimentos. Cada grupo de crianças escolheu dois representantes para a realização da prova, e a montagem ocorreu, simultaneamente, entre meninos e meninas, e todas puderam auxiliar seus representantes. As crianças tiveram dois minutos para completar a pirâmide com os alimentos dispostos no quadro negro.

Na prova do "Semáforo", foram distribuídas as figuras de 22 alimentos para o grupo das meninas e 22 para o grupo dos meninos. Em ambos, havia as mesmas figuras. Em seguida, em uma simulação de semáforo de trânsito montado com isopor, após um sinal vocal descrito como "valendo", cada aluno de cada grupo pegava, aleatoriamente, a figura de um alimento e o distribuía entre as três cores, sendo um grupo por vez. Na parte verde do semáforo, os alunos foram orientados a colocar os alimentos ingeridos frequentemente e em maior quantidade (pães, massas, cereais e tubérculos e o grupo das hortaliças e frutas); na parte amarela, aqueles ingeridos regularmente e em quantidades controladas (carnes, ovos, leites e derivados); e, na parte vermelha, os ingeridos raramente e/ou com moderação (grupo dos açúcares, doces e gorduras). As crianças apenas receberam as informações que, no local verde, deveriam ser colocados os alimentos ingeridos frequentemente e em maior quantidade, sem receber orientações sobre a qual grupo cada um pertencia. Tal orientação também ocorreu para as cores amarela e vermelha.

Havia dois locais distintos na sala com o semáforo para que os alunos (meninas versus meninos) pudessem adicionar os alimentos sem a interferência entre os grupos.

- No encontro 6, as crianças confeccionaram um novo prato para simular uma refeição habitual e saudável (almoço), adotando os conhecimentos adquiridos nas atividades anteriores. Utilizouse a mesma metodologia do encontro 2, para a execução de tal atividade.

- No encontro 7, houve a finalização do estudo com a exposição pareada dos pratos por alunos, em que cada criança demonstrou seu prato denominado de obra de arte antes e depois, lado a lado, para que os demais escolares e outros frequentadores do recinto pudessem analisar a interpretação das crianças quanto à refeição que deveria ser servida em seu almoço. Na figura 2, podem ser observadas as fases de elaboração dos pratos e sua composição de alimentos.

Foi realizado o teste do $\chi 2$ a $5 \%$, de significância, por meio do programa Epi Info, versão 3.5.4, para os dados obtidos na avaliação da percepção dos alimentos pelos sentidos (paladar, tato e visão). Os demais resultados estão apresentados na frequência absoluta e relativa. 
Figura 2. Atividade de elaboração dos pratos. $A$ e $B$ = crianças trabalhando na elaboração dos pratos, $C=$ elaboração dos pratos antes das atividades de educação alimentar e nutricional, $\mathrm{D}$ = elaboração dos pratos depois das atividades de educação alimentar e nutricional. Carmo do Paranaíba, Minas Gerais, Brasil.
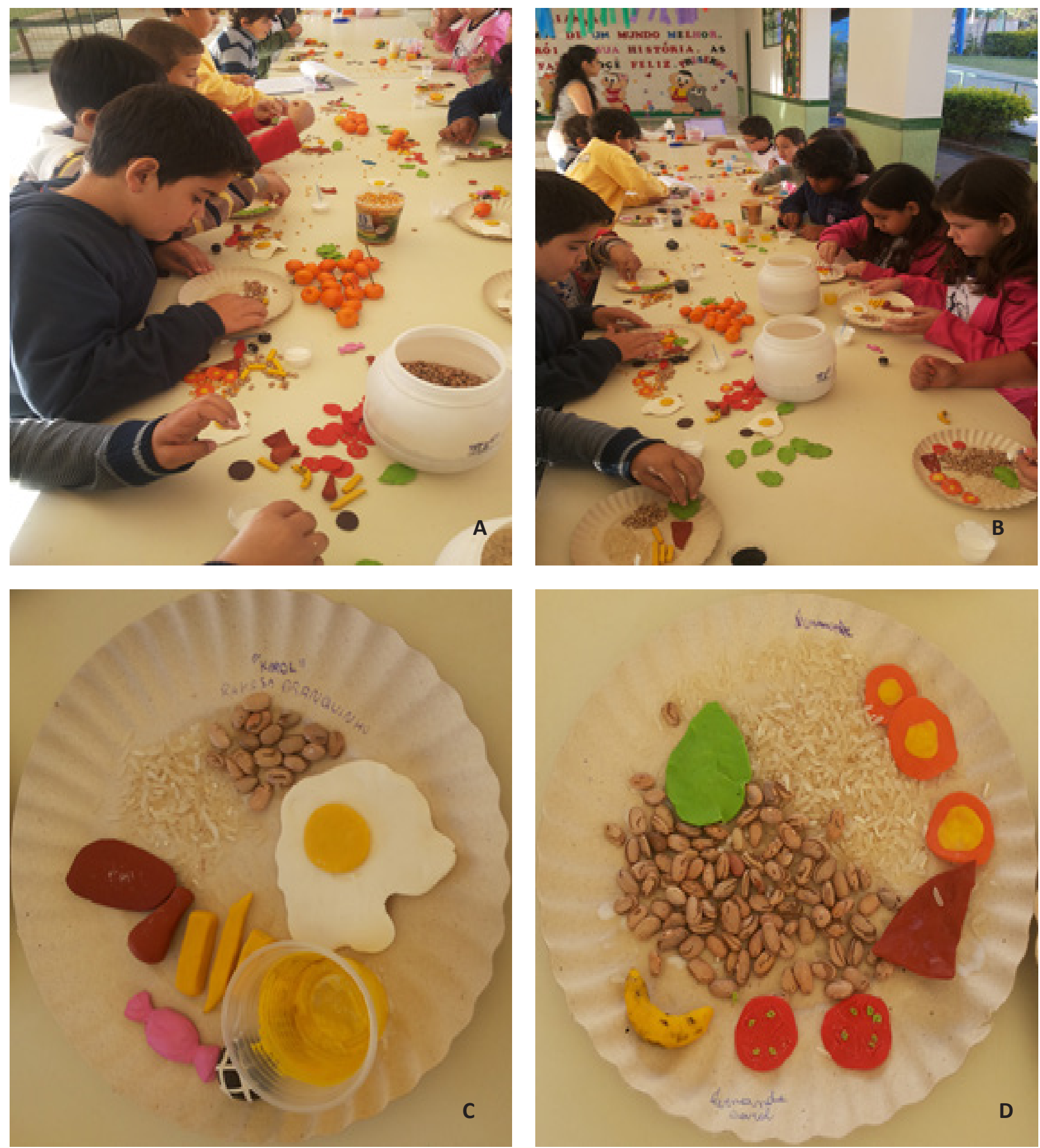


\section{RESULTADOS}

Participaram do estudo 33 crianças, sendo 14 meninos e 19 meninas. No gênero feminino, $36,80 \%(n=7)$ apresentaram sobrepeso e $63,20 \%$ ( $n=12$ ) obesidade, e, no masculino, $28,60 \%$ $(n=4)$ sobrepeso e $71,40 \%(n=10)$ obesidade.

Na dinâmica do "adivinha o que é?", em que foram analisados os alimentos por meio do paladar, tato e visão, observouse, em ambos os gêneros, que o feijão foi o único alimento que apresentou diferença estatística em relação ao tato, demonstrando que as meninas erraram mais o nome desse alimento. O feijão, a cenoura e a banana tiveram $100 \%$ de reconhecimento pela visão. A alface foi bem reconhecida em todos os sentidos avaliados, e, por meio do paladar, apresentou um maior número de erros. A cenoura foi mais reconhecida pelo tato e a visão, porém muitas crianças erraram seu nome na avaliação do paladar. O chuchu apresentou o menor reconhecimento em todos os sentidos avaliados (tabela 1).

Tabela 1. Frequência absoluta dos acertos e erros dos alimentos em relação aos sentidos paladar, tato e visão com as crianças com excesso de peso. Carmo do Paranaíba, Minas Gerais, Brasil.

\begin{tabular}{|c|c|c|c|c|c|c|c|c|c|c|}
\hline \multirow[t]{2}{*}{ Alimento } & \multirow[t]{2}{*}{ Gênero } & \multicolumn{3}{|l|}{ Paladar } & \multicolumn{3}{|l|}{ Tato } & \multicolumn{3}{|l|}{ Visão } \\
\hline & & Acertou & Errou & $\begin{array}{l}\text { Valor } \\
\text { de P }\end{array}$ & Acertou & Errou & $\begin{array}{l}\text { Valor } \\
\text { de P }\end{array}$ & Acertou & Errou & $\begin{array}{l}\text { Valor } \\
\text { de P }\end{array}$ \\
\hline \multirow[t]{3}{*}{ Feijão } & Feminino & 15 & 4 & 1,000 & 11 & 8 & $0,047^{*}$ & 19 & 0 & - \\
\hline & Masculino & 12 & 2 & & 13 & 1 & & 14 & 0 & \\
\hline & Total & 27 & 6 & & 24 & 9 & & 33 & 0 & \\
\hline \multirow[t]{3}{*}{ Alface } & Feminino & 16 & 3 & 1,000 & 16 & 3 & 0,244 & 18 & 1 & 1,000 \\
\hline & Masculino & 12 & 2 & & 14 & 0 & & 14 & 0 & \\
\hline & Total & 28 & 5 & & 30 & 3 & & 32 & 1 & \\
\hline \multirow[t]{3}{*}{ Cenoura } & Feminino & 10 & 9 & 0,723 & 14 & 5 & 1,000 & 19 & 0 & - \\
\hline & Masculino & 9 & 5 & & 11 & 3 & & 14 & 0 & \\
\hline & Total & 19 & 14 & & 25 & 8 & & 33 & 0 & \\
\hline \multirow[t]{3}{*}{ Chuchu } & Feminino & 0 & 19 & 0,067 & 4 & 15 & 0,065 & 8 & 11 & 0,158 \\
\hline & Masculino & 3 & 11 & & 8 & 6 & & 10 & 4 & \\
\hline & Total & 3 & 30 & & 12 & 21 & & 18 & 15 & \\
\hline \multirow[t]{3}{*}{ Banana } & Feminino & 19 & 0 & 0,172 & 17 & 2 & 1,000 & 19 & 0 & - \\
\hline & Masculino & 12 & 2 & & 13 & 1 & & 14 & 0 & \\
\hline & Total & 31 & 2 & & 30 & 3 & & 33 & 0 & \\
\hline
\end{tabular}

*Diferiu estatisticamente a $5 \%$ de significância pelo Teste do $\chi^{2}$.

Em ambos os gêneros, todos os alimentos foram colocados corretamente na pirâmide alimentar, embora os meninos (1 minuto e 07 segundos) tenham realizado tal atividade mais rapidamente que as meninas ( 1 minuto e 12 segundos). Após a atividade, realizou-se uma discussão sobre a importância de cada alimento, e como deve ser o seu consumo habitual para prevenir e tratar doenças, principalmente a obesidade.

$\mathrm{Na}$ prova do semáforo, o gênero masculino apresentou mais erros (tabela 2), principalmente quanto aos alimentos que devem ser ingeridos raramente e/ou com moderação, sugerindo que seu hábito alimentar pode ter influenciado na resposta apresentada.

Tabela 2. Frequência absoluta dos acertos e erros entre os grupos de crianças na prova do semáforo. Carmo do Paranaíba, Minas Gerais, Brasil.

\begin{tabular}{|c|c|c|c|c|c|}
\hline $\begin{array}{l}\text { Gênero e } \\
\text { modalidade de } \\
\text { resposta }\end{array}$ & & & & & Total \\
\hline \multirow[t]{2}{*}{ Feminino } & Acertou & 8 & - & 4 & 12 \\
\hline & Errou & 1 & 3 & 6 & 10 \\
\hline \multirow[t]{2}{*}{ Masculino } & Acertou & 7 & 2 & 5 & 14 \\
\hline & Errou & 2 & 2 & 4 & 8 \\
\hline
\end{tabular}


Após a realização da atividade e da contabilidade dos acertos e erros, ocorreu uma discussão sobre cada alimento e onde ele deveria ser posicionado no semáforo.

Na análise da montagem dos pratos, o feijão permaneceu no prato de todos os avaliados depois das atividades trabalhadas, havendo a retirada do arroz do prato das crianças obesas e dos meninos com sobrepeso, justificando outras atividades de educação alimentar e nutricional com esse público, uma vez que este cereal é importante para tal fase da vida, e poderia ter sido reduzido ou trocado por outro alimento rico em carboidratos. Algumas crianças obesas retiraram as carnes e os ovos de sua refeição elaborada, um fato preocupante visto que esses alimentos são fontes de proteínas, vitaminas e minerais importantes para seu desenvolvimento físico (tabela 3).

Várias crianças retiraram as frutas e as hortaliças dos pratos; entretanto, algumas permaneceram com os alimentos ricos em óleos e gorduras, açúcares e doces, demonstrando a necessidade de outros trabalhos para a fundamentação de conhecimentos que poderão definir seus hábitos alimentares. A maioria dos avaliados adicionaram alimentos doces e/ou ricos em açúcares em seus pratos, sugerindo que a preferência por esse sabor tenha prevalecido diante das informações recebidas sobre a sua qualidade nutricional.

Tabela 3. Frequência relativa (\%) de crianças que apresentaram os alimentos no prato depois das atividades de educação alimentar e nutricional. Carmo do Paranaíba, Minas Gerais, Brasil.

\begin{tabular}{lllrrrrrrr}
\hline $\begin{array}{l}\text { Classificação } \\
\text { nutricional }\end{array}$ & Gênero & $\begin{array}{l}\text { Alimento } \\
\text { no prato }\end{array}$ & Leguminosas & Cereais & $\begin{array}{r}\text { Carnes } \\
\text { e ovos }\end{array}$ & Frutas & Hortaliças & $\begin{array}{r}\text { Óleos e } \\
\text { gorduras }\end{array}$ & $\begin{array}{r}\text { Açúcares } \\
\text { e doces }\end{array}$ \\
\hline Sobrepeso & Feminino & Ausente & - & - & - & 28,57 & 14,28 & 28,57 & 28,57 \\
& $\mathbf{n = 7}$ & Presente & 100,00 & 100,00 & 100,00 & 71,43 & 85,72 & 71,43 & 71,43 \\
& Masculino & Ausente & - & 25,00 & - & 25,00 & - & 75,00 & 75,00 \\
& $\mathbf{n = 4}$ & Presente & 100,00 & 75,00 & 100,00 & 75,00 & 100,00 & 25,00 & 25,00 \\
Obesidade & Feminino & Ausente & - & 25,00 & 16,66 & 8,33 & 25,00 & 58,34 & 25,00 \\
& $\mathbf{n = 1 2}$ & Presente & 100,00 & 75,00 & 83,34 & 91,67 & 75,00 & 41,66 & 75,00 \\
& Masculino & Ausente & - & 30,00 & 20,00 & 10,00 & 10,00 & 70,00 & 20,00 \\
& $\mathbf{n}=10$ & Presente & 100,00 & 70,00 & 80,00 & 90,00 & 90,00 & 30,00 & 80,00 \\
\hline
\end{tabular}

Na figura 3, estão demonstrados os pratos elaborados nos encontros 2 e 6 de duas crianças diferentes. No encontro 2, os pratos possuíam refrigerante, doces e batata frita, e, após as atividades de educação alimentar e nutricional, não houve a presença de tais alimentos para esses avaliados, os quais foram substituídos por suco e fruta (banana).

Figura 3. Pratos elaborados antes e depois das atividades de educação alimentar e nutricional pelas crianças A e B. Carmo do Paranaíba, Minas Gerais, Brasil.
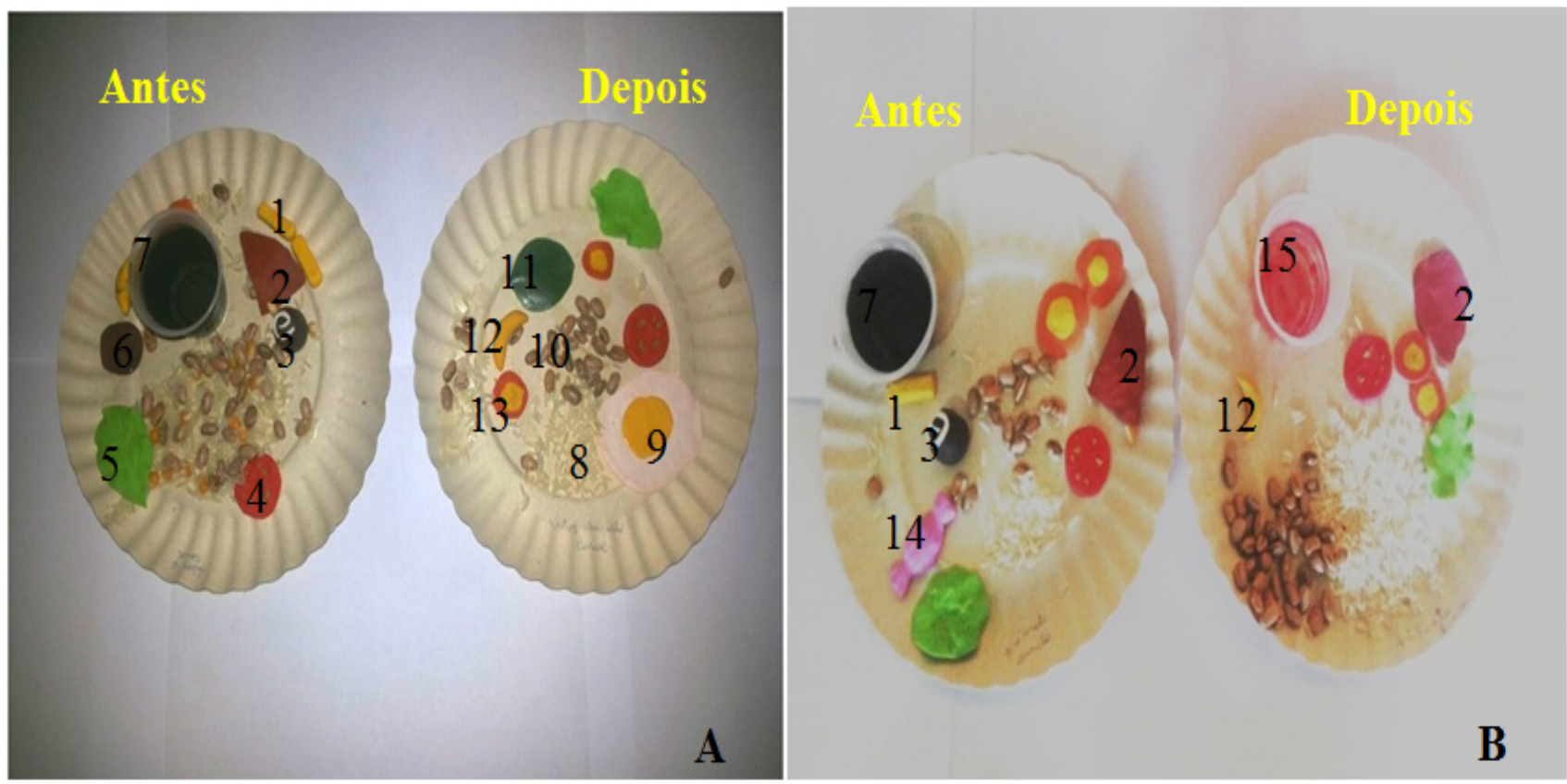

Legenda: 1 - batata-frita; 2 - bife bovino; 3 - bombom; 4 - tomate; 5 - alface; 6 - beterraba; 7 - refrigerante; 8 - arroz; 9 - ovo; 10 - feijão; 11 couve; 12 - banana; 13 - cenoura; 14 - bala; 15 - suco. 
Independentemente do estado nutricional e do gênero, houve uma diminuição da presença do refrigerante e um aumento do suco depois das atividades de educação alimentar e nutricional (tabela 4).
Na figura 4, observa-se que, antes das atividades de educação alimentar e nutricional, havia um grande número de refeições (almoço) com refrigerante, e depois predominou a presença do suco natural.

Tabela 4. Frequência relativa (\%) quanto ao tipo de bebida colocada junto ao prato antes e depois da educação alimentar e nutricional. Carmo do Paranaíba, Minas Gerais, Brasil.

\begin{tabular}{|c|c|c|c|c|c|c|}
\hline Gênero & $\begin{array}{c}\text { Classificação } \\
\text { nutricional }\end{array}$ & $\begin{array}{r}\text { Momento da } \\
\text { avaliação }\end{array}$ & Suco & Refrigerante & Água & $\begin{array}{r}\text { Nenhuma } \\
\text { bebida }\end{array}$ \\
\hline \multirow[t]{4}{*}{ Sobrepeso } & Feminino & Antes & 42,86 & 42,86 & 14,28 & - \\
\hline & $N=7$ & Depois & 71,43 & 28,57 & - & - \\
\hline & Masculino & Antes & - & 100,00 & - & - \\
\hline & $\mathrm{N}=4$ & Depois & 75,00 & - & - & 25,00 \\
\hline \multirow[t]{4}{*}{ Obesidade } & Feminino & Antes & 58,33 & 8,33 & 16,67 & 16,67 \\
\hline & $\mathrm{N}=12$ & Depois & 75,00 & - & 25,00 & - \\
\hline & Masculino & Antes & 40,00 & 30,00 & 20,00 & 10,00 \\
\hline & $\mathrm{N}=10$ & Depois & 50,00 & 20,00 & 30,00 & - \\
\hline
\end{tabular}

Figura 4. Bebidas colocadas junto aos pratos elaborados antes e depois das atividades de educação alimentar e nutricional. Carmo do Paranaíba, Minas Gerais, Brasil.
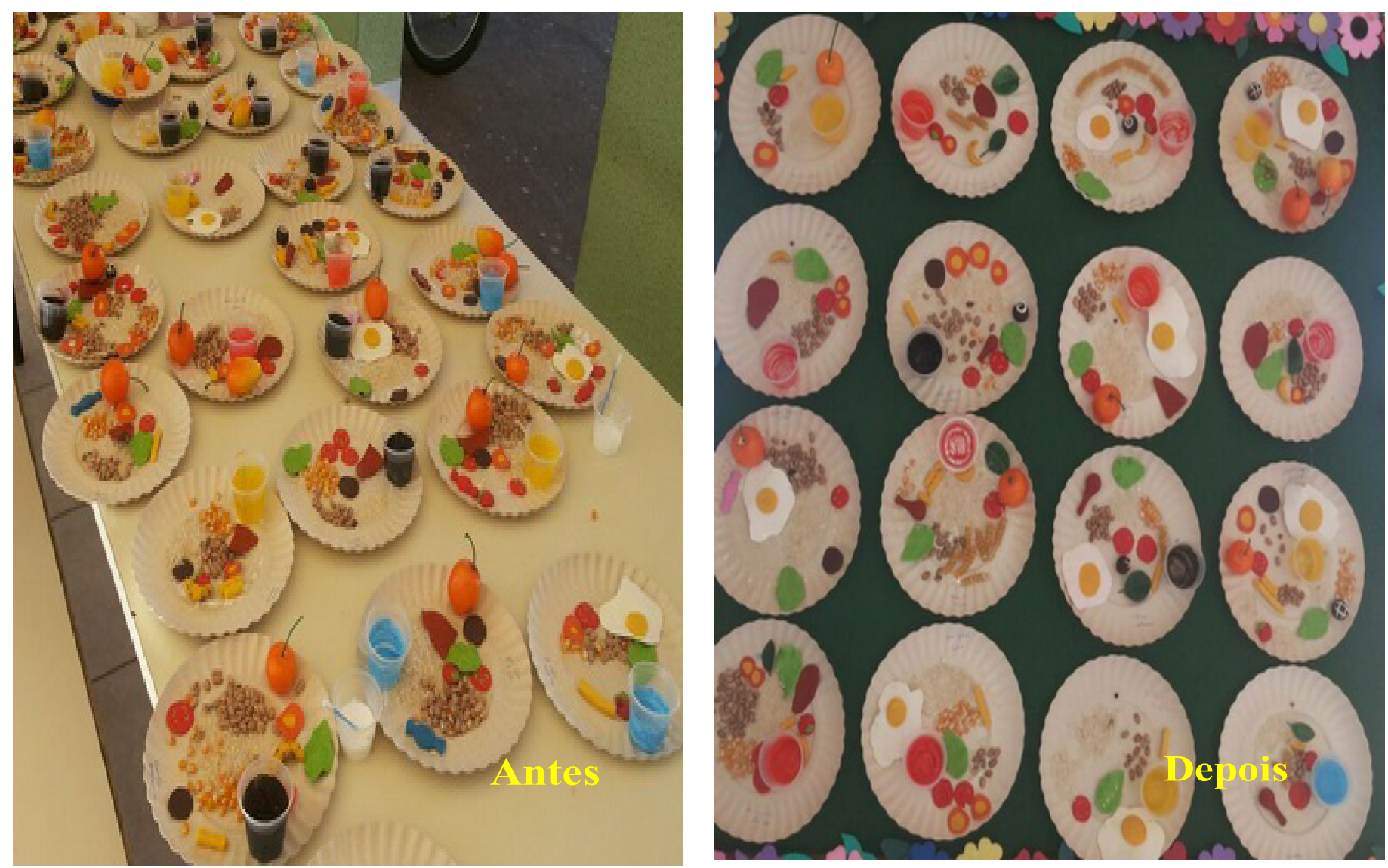

Legenda: 7 - refrigerante; 15 - suco. 


\section{DISCUSSÃO}

Entre as crianças avaliadas, a maioria apresentou obesidade. A obesidade é uma realidade brasileira, principalmente entre o público infantil. Segundo Bertin et al. ${ }^{10}$, as intervenções nutricionais realizadas em crianças são mais eficazes que as mesmas ações na fase adulta e reduzem a gravidade das doenças crônicas oriundas dos maus hábitos alimentares.

O paladar foi o sentido avaliado que obteve mais erros, principalmente em relação ao chuchu em que $90,90 \%$ das crianças não responderam, corretamente, o nome desse alimento. Bezbaruah e Brunt ${ }^{11}$ identificaram que o paladar foi o fator de maior influência para a ingestão de frutas e legumes, destacando que a compreensão de fatores que influenciam o comportamento é essencial para melhorar o consumo de frutas e legumes. O chuchu apresentou o maior número de erro pelos três sentidos avaliados, possivelmente por seu baixo consumo familiar que está associado à sua forma de preparo, sendo que tal alimento é rico em vitaminas, fibras e água e apresenta um baixo custo. Ferarezzi e Costa ${ }^{12}$ destacam que os aspectos sensoriais ditam as escolhas, a aceitação e a preferência, pois suas sensações despertadas definem o hábito alimentar e as escolhas futuras.

A banana apresentou o menor número de erros pelo paladar, possivelmente por seu sabor doce, que é inato ao ser humano, e, segundo a Pesquisa de Orçamentos Familiares ${ }^{13}$, está entre os alimentos mais consumidos pelo brasileiro, sendo parte de sua cultura. De acordo com Birch ${ }^{14}$, a maioria das preferências alimentares é aprendida pelas experiências com a comida e envolve uma condição associativa no contexto social.

Todas as crianças acertaram a posição dos alimentos na pirâmide alimentar. No estudo com escolares do nível fundamental, expostos a um programa de educação alimentar e nutricional, houve um aumento significante no conhecimento sobre nutrição, incluindo a compreensão da pirâmide alimentar, a capacidade de associar os alimentos, seus nutrientes e funções celulares $^{15}$. Segundo Nogueira et al. ${ }^{16}$, os alunos apresentam um maior conhecimento sobre alimentação e nutrição após o desenvolvimento de atividades lúdicas que são prazerosas e inovadoras e podem interferir e colaborar em suas escolhas.

Na prova da montagem do semáforo, a maioria dos erros ocorreu com os alimentos que devem ser ingeridos raramente e/ou com moderação como aqueles ricos em açúcares, os doces e as gorduras. Triches e Giugliani ${ }^{17}$ identificaram que as crianças com sobrepeso e obesidade apresentaram maiores conhecimentos sobre nutrição, sugerindo que tal fato ocorreu pelo interesse em ingerir alimentos mais saudáveis e pelas informações recebidas sobre esse assunto.

Depois das atividades de educação alimentar e nutricional, nos pratos elaborados em ambos os gêneros, houve um aumento na adição de hortaliças e uma redução dos açúcares, doces, refrigerantes e alimentos gordurosos, assim como observado por Gabriel et al. ${ }^{18}$ que identificaram que, antes das atividades de intervenção, as crianças preferiam os salgados fritos e salgadinhos (chips), balas, chicletes, refrigerantes, picolés e sorvetes, e, depois das atividades, aumentou a preferência por sucos naturais e frutas.

No Brasil, dados sobre o consumo alimentar de 26.000 crianças de 5 a 10 anos, oriundos do Sistema de Vigilância Alimentar e Nutricional (SISVAN) (2008), indicaram que 18\% ingeriram biscoitos doces ou recheados, doces, balas e chocolates, nos sete dias de avaliação ${ }^{19}$.

Neste estudo, houve um aumento na adição das frutas nos pratos depois da educação alimentar e nutricional apenas entre as crianças obesas. Segundo Lima et al. ${ }^{20}$, as frutas e as hortaliças, ricas em vitaminas, minerais e água, geralmente não agradam o paladar infantil e são substituídas por outros mais palatáveis que são ricos em açúcares e lipídios, e podem promover distúrbios nutricionais. Coon \& Tucker ${ }^{21}$ destacam sobre a associação direta entre o tempo de assistir televisor e um maior consumo de alimentos calóricos, principalmente os refrigerantes.

Escolares com um melhor conhecimento em nutrição apresentam atitudes e práticas alimentares mais positivas ${ }^{10}$, pois o conhecimento sobre o que comer influencia diretamente no comportamento alimentar saudável ${ }^{22}$.

Geralmente, o público infantil prefere os alimentos que estão disponíveis em casa, e sua aceitação aumenta pela exposição repetida, requerendo de 8 a 10 exposições para as crianças de 2 anos, e de 8 a 15 vezes para aquelas com idade entre 4 a 5 anos $^{23}$. No programa desenvolvido por Psicólogos do Reino Unido para encorajar as crianças a consumirem mais frutas e hortaliças pela degustação repetidamente, a ingestão aumentou em média 0,49 xícaras por dia ${ }^{24}$.

Triches e Giugliani ${ }^{17}$ identificaram que o nível de conhecimentos em nutrição era maior nas crianças obesas, possivelmente porque elas se interessam e são mais passíveis de receber informações sobre o assunto. Porém, elas apresentam práticas alimentares menos saudáveis, considerando seu conhecimento em nutrição. Entretanto, Yokota et al. ${ }^{25}$, ao analisarem crianças das escolas municipais do Rio Grande Sul, observaram que aquelas com um maior Índice de Massa Corporal apresentaram menor conhecimento de nutrição, e possuíam práticas alimentares menos saudáveis. Mesmo relatando conhecer bons hábitos alimentares, os indivíduos obesos, geralmente, não os praticam $^{10}$, pois a obesidade é uma doença multifatorial que está relacionada, principalmente, às mudanças do estilo de vida e dos hábitos alimentares ${ }^{26}$, com destaque para a obesidade dos pais que representa um importante fator de risco para a obesidade infantil'27.

Depois da educação alimentar e nutricional, em ambos os gêneros, houve um aumento na adição de sucos naturais e uma redução dos refrigerantes, assim como observado por Baroni et al. ${ }^{5}$. No Brasil, dados sobre o consumo alimentar de 26.000 crianças de 5 a 10 anos, oriundos do Sistema de Vigilância 
Alimentar e Nutricional (SISVAN), indicaram que $8 \%$ ingeriram refrigerantes nos sete dias de avaliação do estudo ${ }^{19}$, um fato que pode estar contribuindo com o excesso de peso infantil, visto que tais alimentos geralmente são ricos em açúcares simples.

Neste estudo, a maioria das crianças escolheram bebidas mais saudáveis, entretanto algumas permaneceram com hábitos considerados não adequados nutricionalmente. A escola é um ambiente propício para que as ações de educação alimentar e nutricional sejam realizadas ${ }^{5}$. Tais atividades precisam ser inseridas, gradualmente, e necessitam de uma continuidade durante o período escolar ${ }^{10}$, uma vez que as intervenções isoladas não resolvem os problemas oriundos do excesso de peso $^{28}$.

Cecchetto et al. $^{6}$ relatam que mudanças, geralmente, são motivadas a partir de um conhecimento, que é o primeiro passo para empoderar uma criança quanto à importância do cuidar de sua própria saúde. Segundo Souza et al. ${ }^{29}$, as atividades de educação alimentar e nutricional também devem ser voltadas aos funcionários e aos professores, além dos pais ou cuidadores, pois a efetividade do conhecimento adquirido na escola, geralmente, é aplicada no ambiente domiciliar, e pode sofrer influência direta de hábitos já adquiridos pelos pais em situações pregressas. Bernardi et al. ${ }^{30}$ identificaram que, apesar de as principais refeições das crianças terem sido ingeridas na escola; em domicílio, houve um maior consumo de alimentos ricos em carboidratos e lipídios, justificando a necessidade de trabalhos de educação alimentar com os pais ou os cuidadores das crianças.

Como limitação deste estudo, destacam-se o curto período das atividades realizadas, bem como a necessidade de trabalhar, de forma contínua, com as crianças, visto que muitas não reconheceram alguns alimentos, por via dos órgãos dos sentidos avaliados, como também erraram seu posicionamento correto na prova do semáforo. Mesmo depois das atividades de educação alimentar e nutricional, algumas crianças continuaram elaborando pratos não saudáveis.

\section{CONCLUSÃO}

O chuchu foi o alimento menos reconhecido pelos órgãos dos sentidos avaliados, e a banana apresentou o maior número de acertos. A maioria dos erros na prova do semáforo ocorreu com os alimentos que devem ser ingeridos raramente e/ou com moderação. As atividades de educação alimentar e nutricional, lúdicas e artísticas, simples e de baixo custo, melhoraram a percepção quanto à montagem de uma refeição saudável, principalmente em relação ao consumo de açúcares e doces, refrigerantes e alimentos gordurosos.

Faz-se necessária a realização de outros estudos com atividades lúdicas e artísticas, como oficinas culinárias, para prevenir ou tratar o excesso de peso infantil, bem como empoderar esse público para que ele seja um facilitador de mudanças dos hábitos alimentares familiares.

\section{REFERÊNCIAS}

1. Ministério da Saúde (BR), Secretaria de Atenção à Saúde, Departamento de Atenção Básica. Guia alimentar para a população brasileira. 2. ed. Brasília: Ministério da Saúde; 2014. 156p.

2. Instituto Brasileiro de Geografia e Estatística, Coordenação de Trabalho e Rendimento. Pesquisa de orçamentos familiares 2008-2009: antropometria e estado nutricional de crianças, adolescentes e adultos no Brasil. Rio de Janeiro: IBGE; 2010. 127p.

3. Toassa EC, Leal GVS, Wen CL, Philippi ST. Atividades lúdicas na orientação nutricional de adolescentes do Projeto Jovem Doutor. J Brazilian Soc Food Nutr. 2010 Dez; 35(3): 17-27.

4. Hargreaves LM. Alimentarte as obras que alimentam: uma reflexão sobre a produção alimentar artística e seu contexto [dissertação]. Brasília (DF): Universidade de Brasília; 2007.

5. Baroni WSGV, Menezes JBF, Castro SMV. Avaliação da eficiência da educação nutricional em escolares obesos. Nutrivisa. 2017 Nov-Fev; 3(3): 145-154. doi: 10.17648/nutrivisa-vol-3-num-3-g.

6. Cecchetto FH, Pena DB, Pellanda LC. Intervenções lúdicas aumentam o conhecimento sobre hábitos saudáveis e fatores de risco cardiovasculares em crianças: estudo clínico randomizado CARDIOKIDS. Arq Bras Cardiol. 2017; 109(3): 199-206. doi: 10.5935/abc.20170107.

7. Ministério da Saúde (BR). Secretaria de Atenção à Saúde, Departamento de Atenção Básica, Coordenação-Geral da Política de Alimentação e Nutrição. Incorporação das curvas de crescimento da Organização Mundial da Saúde de 2006 e 2007 no SISVAN [Internet]. Brasília: Ministério da Saúde; 2007 [acesso 2018 Jul 21]. Disponível em: http:/nutricao.saude.gov.br/docs/geral/curvas_ oms_2006_2007.pdf.

8. Philippi ST, Cruz ATR, Colucci ACA. Pirâmide alimentar para crianças de 2 a
3 anos. Rev Nutr. 2003 Jan-Mar; 16(1): 5-19. doi: http://dx.doi.org/10.1590/ S1415-52732003000100002.

9. Irala $\mathrm{CH}$, Fernandez PM. Manual para escolas: a escola promovendo hábitos alimentares saudáveis: horta [Internet]. Brasília, 2001 [acesso 2018 Jul 21]. Disponível em: http://bvsms.saude.gov.br/bvs/publicacoes/horta.pdf.

10. Bertin RL, Malkowski J, Zutter LCl, Ulbrich AZ. Estado nutricional, hábitos alimentares e conhecimentos de nutrição em escolares. Rev Paul Pediatr. 2010 Set; 28(3): 303-308. doi: http://dx.doi.org/10.1590/S010305822010000300008.

11. Bezbaruah N, Brunt A. The influence of cartoon character advertising on fruit and vegetable preferences of 9- to 11-year-old children. J Nutr Educ Behav. 2012 Sep; 44(5): 438-441. doi: 10.1016/j.jneb.2011.03.139.

12. Ferarezzi AC, Costa TMB. Análise sensorial. In: Ferarezzi AC, Costa TMB. Nutrição e metabolismo: gestão de qualidade na produção de refeições. Rio de Janeiro: Guanabara Koogan; 2012. p. 231-247.

13. Instituto Brasileiro de Geografia e Estatística, Coordenação de Trabalho e Rendimento. Pesquisa de Orçamentos Familiares 2008-2009: análise do consumo alimentar pessoal no Brasil. Rio de Janeiro: IBGE; 2011. 150 p.

14. Birch LL. Development of food preferences. Annu Rev Nutr. 1999; 19: 41-62.

15. Powers AR, Struempler BJ, Guarino A, Parmer SM. Effects of a nutrition education program on the dietary behavior and nutrition knowledge of secondgrade and third-grade students. J Sch Health. 2005 Apr; 75(4): 129-133.

16. Nogueira CC, Ghisleni CP, Zemolin GP, Zanardo VPS. Perfil nutricional de escolares antes e após educação nutricional. Vivências. 2014 Out; 10(19): 96103. 
17. Triches RM, Giugliani ERJ. Obesidade, práticas alimentares e conhecimentos de nutrição em escolares. Rev Saúde Pública. 2005 Ago; 39(4): 541-547. doi: http://dx.doi.org/10.1590/S0034-89102005000400004.

18. Gabriel CG, Santos MV, Vasconcelos FAG. Avaliação de um programa para promoção de hábitos alimentares saudáveis em escolares de Florianópolis, Santa Catarina, Brasil. Rev Bras Saude Mater Infant. 2008; 8(3): 299-308. doi: http://dx.doi.org/10.1590/S1519-38292008000300009.

19. Ministério da Saúde (BR), Coordenação-Geral da Política de Alimentação e Nutrição Análise dos Registros do Consumo Alimentar. Boletim SISVAN [Internet]. Brasília: Ministério da Saúde. 2009 [acesso 2018 Jul 21]; (10). Disponível em: http://nutricao.saude.gov.br/docs/boletimSisvan/consumo_ alimentar_sisvan_web.pdf.

20. Lima SCVC, Arrais RF, Pedrosa LFC. Avaliação da dieta habitual de crianças e adolescentes com sobrepeso e obesidade. Rev Nutr. 2004; 17(4): 469-477. doi: http://dx.doi.org/10.1590/S1415-52732004000400007.

21. Coon KA, Tucker KL. Television and children's consumption patterns. A review of the literature. Minerva Pediatr. 2002 Oct; 54(5): 423-436.

22. Detregiachi CRP, Braga TMS. Projeto "criança saudável, educação dez": resultados com e sem intervenção do nutricionista. Rev Nutr. 2011; 24(1): 5159. doi: http://dx.doi.org/10.1590/S1415-52732011000100005.

23. Birch LL, Marlin DW. I don't like it; I never tried it: effects of exposure on twoyear-old children's food preference. Appetite. 1982; 3(4): 353-360. doi: https:// doi.org/10.1016/S0195-6663(82)80053-6.

24. Wengreen HJ, Madden GJ, Aguilar SS, Smits RR, Jones BA. Incentivizing children's fruit and vegetable consumption: results of a United States pilot study of the Food Dudes Program. J Nutr Educ Behav. 2013 Jan-Feb; 45(1): 54-59. doi: 10.1016/j.jneb.2012.06.001.

25. Yokota, RTC, Vasconcelos TF, Pinheiro ARO, Schmitz BAS, Coitinho DC, Rodrigues MLCF. Projeto "a escola promovendo hábitos alimentares saudáveis": comparação de duas estratégias de educação nutricional no Distrito Federal, Brasil. Rev Nutr. 2010 Jan-Fev; 23(1): 37-47. doi: http://dx.doi.org/10.1590/ S1415-52732010000100005.

26. Toral N, Slater B, Cintra IP, Fisber M. Comportamento alimentar de adolescentes em relação ao consumo de frutas e verduras. Rev Nutr. 2006 Maio-Jun; 19(3): 331-340. doi: http://dx.doi.org/10.1590/S141552732006000300004.

27. Traebert J, Moreira EAM, Bosco VL, Almeida ICS. Transição alimentar: problema comum à obesidade e à cárie dentária. Rev Nutr. 2004 Jun; 17(2): 247-253. doi: http://dx.doi.org/10.1590/S1415-52732004000200011.

28. Müller MJ, Mast M, Asbeck I, Langnäse K, Grund A. Preventions of obesity is it possible? Obes Rev. 2001 Feb; 2(1): 15-28.

29. Souza SC, Cunha DC, Bissoli MC. Avaliação de intervenção nutricional para pré-escolares de um centro educacional em um município do Sul de Minas Gerais. Rev Cienc Saúde. 2012 Jan; 2(1): 1-8.

30. Bernardi JR, Cezaro C, Fisberg RM, Fisberg M, Vitolo MR. Estimativa do consumo de energia e de macronutrientes no domicílio e na escola em préescolares. J Pediatr. 2010 Jan-Fev; 86(1): 59-64. doi: http://dx.doi.org/10.1590/ S0021-75572010000100011.

\section{Como citar este artigo/How to cite this article:}

Vinhal CA, Lisboa LCO, Almeida MEF. Atividade artística para avaliar o consumo alimentar e os conhecimentos de nutrição em crianças com excesso de peso . J Health Biol Sci. 2019 Out-Dez; 7(4):364-373. 